\title{
Feaftures of the double photoionization spectra of two-electron impurity centers in quasi-zero-dimensional structures
}

\author{
V. D. Krevchik, A. V. Razumov, P. S. Budyansky, M. B. Semenov, I. M. Moyko \\ Penza State University, Krasnaya str., 40, Penza, 440026, Russia \\ physics@pnzgu.ru
}

PACS 73.40.Gk, 03.65.Xp

DOI 10.17586/2220-8054-2019-10-5-540-548

\begin{abstract}
The zero-range potential method has been generalized to the case of two-electron impurity centers with an effective nuclear charge equal to zero in a spherically symmetric quantum dot (QD), and on the basis of this method the first ionization potential has been calculated by variational method. It is shown that as the radius of QD decreases, the threshold value of the second ionization potential also decreases, beginning with which the existence of the two-electron bound state is possible due to an increase in the size-quantization energy accompanied by suppression of mutual electron repulsion. The light impurity absorption coefficient has been calculated using the dipole approximation for double ionization of the twoelectron impurity center by a single photon in a quasi-zero-dimensional structure, which is the transparent dielectric matrix with semiconductor QDs synthesized in it. It is shown that characteristic feature of the double photoionization spectrum is a two-humped profile of the spectral curve due to electron correlations.
\end{abstract}

Keywords: double photoionization spectra, two-electron impurity centers, quasi-zero-dimensional structures.

Received: 5 September 2019

Revised: 18 September 2019

\section{Introduction}

A large number of impurities in semiconductors in the bound state can possess not only one, but also two electrons, i.e. these impurities are the helium-like impurity centers [1-14]. Difficulties in the theoretical study of the two-electron impurity states are due to the complex character of the interaction between electrons from the outer shell of the impurity center with valence electrons of the nearest lattice atoms [1]. As a result, it turns out to be impossible to predict not only the position of the multiply charged centers levels, but also their possible charge states. In [1], a variational method has been used to calculate the ground state of the two-electron impurity center. Simulation of the two-electron impurity was based on generalization to the case of two electrons for the Lukovsky model [2]. As is known within the framework of this model, it is assumed that there is a short-range potential of zero radius which determines the ionization energy of a singly ionized impurity, i.e. the second ionization potential, $E_{2}$. The authors [1] succeeded in calculating of the first ionization potential $E_{1}$ as a function of the nucleus charge $Z$ for the impurity center, with the second ionization potential being taken as the empirical parameter. The process of double photoionization for twoelectron impurity centers by a single photon is of special interest. This reaction is one of the fundamental reactions involving several particles. The present paper is devoted to calculation of the first ionization potential for the twoelectron impurity center in QD by the variational method, as well as the theoretical study of features of the double photoionization spectra of two-electron impurity centers in a quasi-zero-dimensional structure.

\section{Relationship between the first and second ionization potentials of the two-electron impurity center in quantum dot. Comparison with the bulk semiconductor case}

We use the semi-empirical model of two-electron impurity centers developed in [1]. The short-range potential in this model is approximated by a potential well of depth $V_{0}$, radius of $d$ which is much smaller than the radius of the localized state. As an empirical parameter, we take the energy of a doubly ionized impurity, i.e. the second ionization potential $E_{2}$.

Thus, the task is to calculate the first ionization potential $E_{1}$. The value $E_{1}$ can be found by the variational method with wave functions taken in the form of the product of one-electron wave functions $\Psi\left(\rho_{i}\right)$ with a variational parameter $\eta$, which is associated with the variable energy of the one-electron state.

As units of length and energy, we choose, respectively $a_{d}=\varepsilon \hbar^{2} /\left(m^{*} e^{2}\right)$ - the effective Bohr radius and $E_{d}=$ $\hbar^{2} /\left(2 m^{*} a_{d}^{2}\right)$ - the effective Bohr energy. In this case, we take into account that the power of the short-range potential $V_{0} d^{2}$ at $d \rightarrow 0$ remains finite. The two-electron wave function satisfies the Schrödinger equation:

$$
\mathbf{H}\left(\rho_{1}, \rho_{2}\right) \Psi\left(\rho_{1}, \rho_{2}\right)=E \Psi\left(\rho_{1}, \rho_{2}\right),
$$


where

$$
\begin{gathered}
\mathbf{H}\left(\rho_{1}, \rho_{2}\right)=\mathbf{H}\left(\rho_{1}\right)+\mathbf{H}\left(\rho_{2}\right)+\hbar^{2} /\left(m^{*} a_{d}^{2}\left|\vec{\rho}_{1}-\vec{\rho}_{2}\right|\right), \\
\mathbf{H}\left(\rho_{i}\right)=-\hbar^{2} \Delta_{i} /\left(2 m^{*} a_{d}^{2}\right)-\hbar^{2} V\left(\rho_{i}\right) /\left(2 m^{*} a_{d}^{2}\right), \\
\rho_{d}=d / a_{d}, \quad V\left(\rho_{i}\right)=\left\{\begin{array}{ll}
V_{0}^{*}, & \rho_{i} \leq \rho_{d} ; \\
0, & \rho_{i}>\rho_{d},
\end{array}, \quad V_{0}^{*}=V_{0} / E_{d}, \quad \rho_{i}=r_{i} / a_{d},\right.
\end{gathered}
$$

$r_{i}$ - electron coordinates at $i=1,2, m^{*}$ - effective electron mass.

Usually, the simplest form of a two-electron wave function $\Psi\left(\rho_{1}, \rho_{2}\right)$ leading to a satisfactory approximation in problems of helium-like centers is the product of one-electron wave functions:

$$
\Psi\left(\rho_{1}, \rho_{2}\right)=\Psi\left(\rho_{1}\right) \Psi\left(\rho_{2}\right)
$$

Using the results of [5], where in the framework of the spherically symmetric potential well model (the "hard wall" model), an expression is obtained for the wave function of an electron localized at a short-range potential in a quantum dot, and for the one-electron wave function $\Psi\left(\rho_{i}\right)$ we have:

$$
\Psi\left(\rho_{i}\right)=\frac{B}{\rho_{i}} \begin{cases}\frac{\sinh \left(R_{0}^{*} \eta^{-1}-\rho_{d} \eta^{-1}\right)}{\sinh \left(R_{0}^{*} \eta^{-1}\right)} \frac{\sin \left(\chi_{0} \rho_{i}\right)}{\sin \left(\chi_{0} \rho_{d}\right)}, & \rho_{i} \leq \rho_{d} ; \\ \frac{\sinh \left(R_{0}^{*} \eta^{-1}-\rho_{i} \eta^{-1}\right)}{\sinh \left(R_{0}^{*} \eta^{-1}\right)}, & \rho_{i} \geq \rho_{d},\end{cases}
$$

here, $\chi_{0}=\sqrt{V_{0}^{*}-\eta^{-2}}, V_{0}^{*}=V_{0} / E_{d}, \eta=\sqrt{E_{d} /\left|E_{2}\right|}, R_{0}^{*}=R_{0} / a_{d}, R_{0}-$ the QD radius,

$$
B=\sqrt{\frac{2 \eta^{-1}}{\tanh \left(R_{0}^{*} \eta^{-1}\right)-R_{0}^{*} \eta^{-1} \csc \left(R_{0}^{*} \eta^{-1}\right)}} .
$$

Taking into account (3), the test two-electron wave function is written in the next form:

$$
\Psi\left(\rho_{1}, \rho_{2}\right)=\frac{B^{2}}{\rho_{1} \rho_{2}} \begin{cases}\frac{\sinh ^{2}\left(R_{0}^{*} \eta^{-1}-\rho_{d} \eta^{-1}\right)}{\sinh ^{2}\left(R_{0}^{*} \eta^{-1}\right)} \frac{\sin \left(\chi_{0} \rho_{1}\right) \sin \left(\chi_{0} \rho_{2}\right)}{\sin ^{2}\left(\chi_{0} \rho_{d}\right)}, & \rho_{i} \leq \rho_{d} ; \\ \frac{\sinh \left(R_{0}^{*} \eta^{-1}-\rho_{1} \eta^{-1}\right) \sinh \left(R_{0}^{*} \eta^{-1}-\rho_{2} \eta^{-1}\right)}{\sinh ^{2}\left(R_{0}^{*} \eta^{-1}\right)}, & \rho_{i} \geq \rho_{d} .\end{cases}
$$

The energy of a two-electron impurity center $\varepsilon\left(R_{0}^{*}, \eta\right)$ is determined by the minimum of the average value of the Hamiltonian:

$$
\frac{\varepsilon\left(R_{0}^{*}, \eta\right)}{E_{d}}=\frac{\left\langle\Psi\left(\rho_{1}, \rho_{2}\right)\left|\mathbf{H}\left(\rho_{1}, \rho_{2}\right)\right| \Psi\left(\rho_{1}, \rho_{2}\right)\right\rangle}{\left\langle\left|\Psi\left(\rho_{1}, \rho_{2}\right)\right|^{2}\right\rangle}
$$

Expression (5), taking into account (4), can be represented in the form: 


$$
\begin{aligned}
& \frac{\varepsilon\left(R_{0}^{*}, \eta\right)}{E_{d}}= \\
& -\frac{2^{6} \eta^{-2} \pi^{2}}{\sinh ^{4}\left(R_{0}^{*} \eta^{-1}\right)\left(\tanh \left(R_{0}^{*} \eta^{-1}\right)-R_{0}^{*} \eta^{-1} \csc \left(R_{0}^{*} \eta^{-1}\right)\right)^{2}}\left\{\frac{\sinh ^{4}\left(R_{0}^{*} \eta^{-1}-\rho_{d} \eta^{-1}\right)}{\sin ^{4}\left(\chi_{0} \rho_{d}\right)} \times\right. \\
& {\left[\frac{\eta^{-2}}{16 \chi_{0}^{2}}\left(\sin \left(2 \chi_{0} \rho_{d}\right)-2 \chi_{0} \rho_{d}\right)^{2}-\frac{\left(4 \chi_{0} \rho_{d}-2 \sin \left(2 \chi_{0} \rho_{d}\right)-2 \operatorname{Si}\left(2 \chi_{0} \rho_{d}\right)+\operatorname{Si}\left(4 \chi_{0} \rho_{d}\right)\right)^{2}}{4 \chi_{0}}\right]+} \\
& 2^{-4}\left(2 \eta^{-1}\left(R_{0}^{*}-\rho_{d}\right)-\sinh \left(2 \eta^{-1}\left(R_{0}^{*}-\rho_{2}\right)\right)\right)^{2}- \\
& \frac{\eta}{2^{3}}\left\{4 \eta^{-1}\left(R_{0}^{*}-\rho_{d}\right)-4 \eta^{-1} \rho_{d} \ln \left(\frac{R_{0}^{*}}{\rho_{d}}\right)-\sinh \left(4 R_{0}^{*} \eta^{-1}\right)\left[\operatorname{Chi}\left(4 R_{0}^{*} \eta^{-1}\right)-\operatorname{Chi}\left(4 \rho_{d} \eta^{-1}\right)\right]-\right. \\
& \sinh \left(4 \eta^{-1}\left(R_{0}^{*}-\rho_{d}\right)\right)-2 \sinh \left(4 \eta^{-1}\left(R_{0}^{*}-\rho_{d}\right)\right) \ln \left(\frac{R_{0}^{*}}{\rho_{d}}\right)+2\left[\operatorname{Chi}\left(4 \eta^{-1} R_{0}^{*}\right)-\operatorname{Chi}\left(4 \eta^{-1} \rho_{d}\right)\right] \times \\
& {\left[\sinh \left(2 \eta^{-1} R_{0}^{*}\right)+\cosh \left(2 \eta^{-1} R_{0}^{*}\right)\left(2 \eta^{-1} \rho_{d}+\sinh \left(2 \eta^{-1}\left(R_{0}^{*}-\rho_{d}\right)\right)\right)\right]-} \\
& \cosh \left(2 \eta^{-1} R_{0}^{*}\right)\left[\operatorname{Shi}\left(2 \eta^{-1} R_{0}^{*}\right)-\operatorname{Shi}\left(2 \eta^{-1} \rho_{d}\right)\right]+\cosh \left(2 \eta^{-1} \rho_{d}\right) \times \\
& {\left[\operatorname{Shi}\left(2 \eta^{-1} R_{0}^{*}\right)-\operatorname{Shi}\left(2 \eta^{-1} \rho_{d}\right)\right]-\cosh \left(2 \eta^{-1}\left(2 R_{0}^{*}-\rho_{d}\right)\right)\left[\operatorname{Shi}\left(2 \eta^{-1} R_{0}^{*}\right)-\operatorname{Shi}\left(2 \eta^{-1} \rho_{d}\right)\right]-} \\
& 4 \eta^{-1} \rho_{d} \sinh \left(2 \eta^{-1} R_{0}^{*}\right)\left[\operatorname{Shi}\left(2 \eta^{-1} R_{0}^{*}\right)-\operatorname{Shi}\left(2 \eta^{-1} \rho_{d}\right)\right]+ \\
& \left.\left.\cosh \left(4 \eta^{-1} R_{0}^{*}\right) \times\left[\operatorname{Shi}\left(4 \eta^{-1} R_{0}^{*}\right)-\operatorname{Shi}\left(4 \eta^{-1} \rho_{d}\right)\right]\right\}\right\},
\end{aligned}
$$

where Chi $(x)$ and Shi $(x)$ - integral hyperbolic cosine and sine, respectively.

To perform transition to the limit, it is necessary to study the behavior of the short-range potential power $\gamma=\rho_{d}^{2} \chi_{0}^{2}$ at $d \rightarrow 0$. For this purpose, we use the continuity of the derivative of the one-electron wave function (3) at $\rho_{i}=\rho_{d}$. Elementary calculations lead to the following:

$$
\cos \left(\rho_{d} \chi_{0}\right)=0
$$

In the limit $d \rightarrow 0$, we have:

$$
\gamma=\left(\rho_{d}^{2} \chi_{0}^{2}\right)_{d \rightarrow 0}=\left[\frac{\pi}{2}(2 n+1)\right]^{2}, \quad n=0,1,2 \ldots
$$

Thus, the value $\gamma$ can take only discrete values defined by the expression (8).

Condition (8) allows one to obtain the limiting values of some trigonometric and integral functions that are necessary for the transition to the limit:

$$
\begin{aligned}
\sin \left(\chi_{0} \rho_{d}\right)=0, & \sin \left(2 \chi_{0} \rho_{d}\right)=0, & & \operatorname{Si}\left(2 \chi_{0} \rho_{d}\right)=0, \\
\operatorname{Si}\left(4 \chi_{0} \rho_{d}\right)=0, & \operatorname{Chi}\left(4 \rho_{d} \eta^{-1}\right)=1, & & \operatorname{Shi}\left(2 \eta^{-1} \rho_{d}\right)=0 .
\end{aligned}
$$

Having made the transition to the limit $(d \rightarrow 0)$ in (6), taking into account (9), for $\varepsilon\left(R_{0}^{*}, \eta\right) / E_{d}$ we obtain:

$$
\begin{aligned}
& \frac{\varepsilon\left(R_{0}^{*}, \eta\right)}{E_{d}}= \\
& -2^{3} \eta^{-2} \pi^{2} \sinh ^{-4}\left(R_{0}^{*} \eta^{-1}\right)\left(\tanh \left(R_{0}^{*} \eta^{-1}\right)-R_{0}^{*} \eta^{-1} \csc \left(R_{0}^{*} \eta^{-1}\right)\right)^{-2} \times \\
& \left\{2^{-1}\left(2 \eta^{-1} R_{0}^{*}-\sinh \left(2 \eta^{-1} R_{0}^{*}\right)\right)^{2}+\eta\left[-4 \eta^{-1} R_{0}^{*}-8 \cosh ^{3}\left(\eta^{-1} R_{0}^{*}\right) \sinh \left(\eta^{-1} R_{0}^{*}\right) \operatorname{Chi}\left(2 \eta^{-1} R_{0}^{*}\right)+\right.\right. \\
& 2 \eta^{-1} R_{0}^{*}+2 \ln \left(2 \exp (1+C) \eta^{-1} R_{0}^{*}\right) \sinh \left(2 \eta^{-1} R_{0}^{*}\right)+\left(\operatorname{Chi}\left(4 \eta^{-1} R_{0}^{*}\right)-\ln 2\right) \sinh \left(4 \eta^{-1} R_{0}^{*}\right)- \\
& \left.\left.\operatorname{Shi}\left(2 \eta^{-1} R_{0}^{*}\right)\left(1-2 \cosh \left(2 \eta^{-1} R_{0}^{*}\right)-\cosh \left(4 \eta^{-1} R_{0}^{*}\right)\right)+\cosh \left(4 \eta^{-1} R_{0}^{*}\right) \operatorname{Shi}\left(4 \eta^{-1} R_{0}^{*}\right)\right]\right\}
\end{aligned}
$$

here, $C=0.577-$ the Euler constant. 
Minimization with respect to the parameter $\eta$ leads to a transcendental equation for finding the extreme value $\tilde{\eta}$ :

$$
\frac{\partial \varepsilon\left(R_{0}^{*}, \eta\right)}{\partial \eta}=0
$$

The explicit expression for (11) has the following:

$$
\begin{aligned}
& \left\{\frac{2^{7} \pi^{2} \tilde{\eta}^{-2}}{\sinh ^{4}\left(R_{0}^{*} \tilde{\eta}^{-1}\right)\left(\tanh \left(R_{0}^{*} \tilde{\eta}^{-1}\right)-R_{0}^{*} \tilde{\eta}^{-1} \csc \left(R_{0}^{*} \tilde{\eta}^{-1}\right)\right)^{3}} \times\right. \\
& {\left[R_{0}^{*} \tilde{\eta}^{-2} \csc \left(2 \eta^{-1} R_{0}^{*}\right)-R_{0}^{*} \tilde{\eta}^{-3} \cot \left(2 \tilde{\eta}^{-1} R_{0}^{*}\right) \csc \left(2 \tilde{\eta}^{-1} R_{0}^{*}\right)-R_{0}^{*} \tilde{\eta}^{-2} \operatorname{sech}\left(2 \tilde{\eta}^{-1} R_{0}^{*}\right)\right]+} \\
& \left.\frac{2^{7} \pi^{2} \tilde{\eta}^{-3}-2^{8} \pi^{2} \tilde{\eta}^{-4} \operatorname{coth}\left(R_{0}^{*} \tilde{\eta}^{-1}\right)}{\sinh ^{4}\left(R_{0}^{*} \tilde{\eta}^{-1}\right)\left(\tanh \left(R_{0}^{*} \tilde{\eta}^{-1}\right)-R_{0}^{*} \tilde{\eta}^{-1} \csc \left(R_{0}^{*} \tilde{\eta}^{-1}\right)\right)^{2}}\right\} \times \\
& \left\{\frac{1}{16}\left(2 R_{0}^{*} \tilde{\eta}^{-1}-\sinh \left(2 \tilde{\eta}^{-1} R_{0}^{*}\right)\right)^{2}+\frac{1}{8} \tilde{\eta}\left[-4 R_{0}^{*} \tilde{\eta}^{-1}-8 \cosh ^{3}\left(2 \tilde{\eta}^{-1} R_{0}^{*}\right) \operatorname{Ci}\left(2 \tilde{\eta}^{-1} R_{0}^{*}\right) \sinh \left(\tilde{\eta}^{-1} R_{0}^{*}\right)+\right.\right. \\
& 2 \ln \left(\tilde{\eta}^{-1} R_{0}^{*} e^{1+\gamma}\right) \sinh \left(2 \tilde{\eta}^{-1} R_{0}^{*}\right)+\left(\operatorname{Ci}\left(4 \tilde{\eta}^{-1} R_{0}^{*}\right)-\ln 2\right) \sinh \left(4 \tilde{\eta}^{-1} R_{0}^{*}\right)-\operatorname{Si}\left(2 \tilde{\eta}^{-1} R_{0}^{*}\right) \times \\
& \left.\left.\left(1-2 \cosh \left(2 \tilde{\eta}^{-1} R_{0}^{*}\right)-\cosh \left(4 \tilde{\eta}^{-1} R_{0}^{*}\right)\right)-\operatorname{Si}\left(4 \tilde{\eta}^{-1} R_{0}^{*}\right)+\cosh \left(4 \tilde{\eta}^{-1} R_{0}^{*}\right) \operatorname{Si}\left(4 \tilde{\eta}^{-1} R_{0}^{*}\right)\right]\right\}- \\
& \frac{2^{6} \pi^{2} \tilde{\eta}^{-2}}{\sinh ^{4}\left(R_{0}^{*} \tilde{\eta}^{-1}\right)\left(\tanh \left(R_{0}^{*} \tilde{\eta}^{-1}\right)-R_{0}^{*} \tilde{\eta}^{-1} \csc \left(R_{0}^{*} \tilde{\eta}^{-1}\right)\right)^{2}} \times \\
& \left\{\left[\frac{1}{8}\left(2 R_{0}^{*} \tilde{\eta}^{-2}-2 \tilde{\eta}^{-2} \cosh \left(2 \tilde{\eta}^{-1} R_{0}^{*}\right)\right)^{2}\left(2 R_{0}^{*} \tilde{\eta}^{-1}-\sinh \left(2 \tilde{\eta}^{-1} R_{0}^{*}\right)\right)+\right.\right. \\
& \frac{\tilde{\eta}}{8}\left[4 R_{0}^{*} \tilde{\eta}^{-2}+8 \tilde{\eta}^{-2} R_{0}^{*} \cosh ^{4}\left(\tilde{\eta}^{-1} R_{0}^{*}\right) \mathrm{Ci}\left(2 \tilde{\eta}^{-1} R_{0}^{*}\right)+4 \tilde{\eta}^{-2} R_{0}^{*} \cosh ^{4}\left(4 \tilde{\eta}^{-1} R_{0}^{*}\right)+\right. \\
& 2 \ln \left(\tilde{\eta}^{-1} R_{0}^{*} e^{1+\gamma}\right) \sinh \left(2 \tilde{\eta}^{-1} R_{0}^{*}\right)+\left(\mathrm{Ci}\left(4 \tilde{\eta}^{-1} R_{0}^{*}\right)-\ln 2\right) \sinh \left(4 \tilde{\eta}^{-1} R_{0}^{*}\right)-\operatorname{Si}\left(2 \tilde{\eta}^{-1} R_{0}^{*}\right) \times \\
& \left.\left.\left(1-2 \cosh \left(2 \tilde{\eta}^{-1} R_{0}^{*}\right)-\cosh \left(4 \tilde{\eta}^{-1} R_{0}^{*}\right)\right)-\operatorname{Si}\left(4 \tilde{\eta}^{-1} R_{0}^{*}\right)+\cosh \left(4 \tilde{\eta}^{-1} R_{0}^{*}\right) \operatorname{Si}\left(4 \tilde{\eta}^{-1} R_{0}^{*}\right)\right]\right]+ \\
& \frac{1}{8}\left[-4 R_{0}^{*} \tilde{\eta}^{-1}-8 \cosh ^{3}\left(2 \tilde{\eta}^{-1} R_{0}^{*}\right) \operatorname{Ci}\left(2 \tilde{\eta}^{-1} R_{0}^{*}\right) \sinh \left(\tilde{\eta}^{-1} R_{0}^{*}\right) \times\right. \\
& \left(\mathrm{Ci}\left(2 \tilde{\eta}^{-1} R_{0}^{*}\right)-\ln 2\right)-4 \tilde{\eta}^{-2} R_{0}^{*} \ln \left(\tilde{\eta}^{-1} R_{0}^{*} e^{1+\gamma}\right) \cosh \left(2 \tilde{\eta}^{-1} R_{0}^{*}\right)+8 \tilde{\eta}^{-1} \cosh ^{3}\left(\tilde{\eta}^{-1} R_{0}^{*}\right) \times \\
& \cosh \left(2 \tilde{\eta}^{-1} R_{0}^{*}\right) \sinh \left(\tilde{\eta}^{-1} R_{0}^{*}\right)+24 \tilde{\eta}^{-1} R_{0}^{*} \cosh ^{2}\left(\tilde{\eta}^{-1} R_{0}^{*}\right) \operatorname{Ci}\left(2 \tilde{\eta}^{-1} R_{0}^{*}\right) \sinh ^{2}\left(\tilde{\eta}^{-1} R_{0}^{*}\right)- \\
& 2 \tilde{\eta}^{-1} \sinh \left(2 \tilde{\eta}^{-1} R_{0}^{*}\right)-\tilde{\eta}^{-1} \sinh \left(2 \tilde{\eta}^{-1} R_{0}^{*}\right)\left(1-2 \cosh \left(2 \tilde{\eta}^{-1} R_{0}^{*}\right)-\cosh \left(4 \tilde{\eta}^{-1} R_{0}^{*}\right)\right)+ \\
& \tilde{\eta}^{-1} \sinh \left(4 \tilde{\eta}^{-1} R_{0}^{*}\right)-2 \tilde{\eta}^{-1} \cosh \left(4 \tilde{\eta}^{-1} R_{0}^{*}\right) \sinh \left(4 \tilde{\eta}^{-1} R_{0}^{*}\right)-4 \tilde{\eta}^{-1} R_{0}^{*} \operatorname{Si}\left(2 \tilde{\eta}^{-1} R_{0}^{*}\right) \times \\
& \left.\left.\left(\sinh \left(4 \tilde{\eta}^{-1} R_{0}^{*}\right)+\sinh \left(4 \tilde{\eta}^{-1} R_{0}^{*}\right)\right)-4 \tilde{\eta}^{-1} R_{0}^{*} \sinh \left(4 \tilde{\eta}^{-1} R_{0}^{*}\right) \operatorname{Si}\left(4 \tilde{\eta}^{-1} R_{0}^{*}\right)\right]\right\}=0 .
\end{aligned}
$$

Taking into account that the minimum value of the functional $\varepsilon\left(R_{0}^{*}, \eta\right) / E_{d}$ achieved with the extreme value of the parameter $\eta=\tilde{\eta}$ is the sum:

$$
\frac{\varepsilon\left(R_{0}^{*}, \eta\right)}{E_{d}}=-\frac{E_{1}}{E_{d}}-\frac{E_{2}}{E_{d}},
$$

one can find the first ionization potential $E_{1}$ as a function of the second ionization potential $E_{2}$ taken from the experiment:

$$
\frac{E_{1}}{E_{d}}=-\frac{\varepsilon\left(R_{0}^{*}, \eta\right)}{E_{d}}-\frac{E_{2}}{E_{d}} .
$$

Figure 1 shows relationship between the first and second ionization potentials of the two-electron impurity center with the nucleus zero charge $(Z=0)$ in a semiconductor QD in the Bohr energy units, obtained by numerical calculations (curves 1,2,3), and also in the bulk semiconductor case (curves 4, 5,6) [1]. 


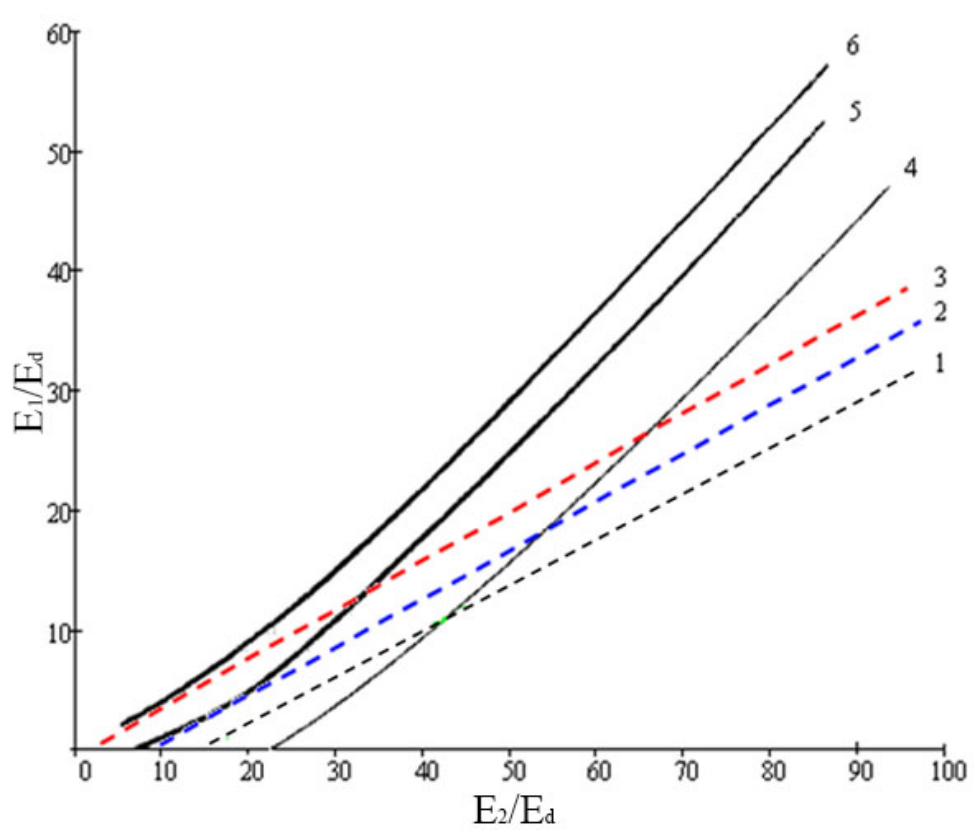

FIG. 1. Connection between the first and second ionization potentials of the two-electron impurity center with the nucleus zero charge $(Z=0)$ (curves $1,2,3)$ in a semiconductor QD and for the bulk semiconductor case [1] (curves $4,5,6$ ), for different values of $R_{0}^{*}$ :

1: $R_{0}^{*}=0.5 ; 2: R_{0}^{*}=1 ; 3: R_{0}^{*}=3$ and for different values of the nucleus charges $Z$ for the two-electron impurity center: $4: Z=1 ; 5: Z=2 ; 6: Z=3$

It can be seen that as the QD radius $R_{0}^{*}$ increases, the threshold energy $E_{2} / E_{d}$, beginning with which the existence of the two-electron bound state is possible, also increases. This is due to the fact that energy of the electrons' mutual repulsion is not compensated for by the corresponding Coulomb attraction to the nucleus, since $Z=0$. Indeed, the action of the short-range potential is sharply attenuated due to an increase in the electrons' localization effective radius because of their repulsion. With decreasing $R_{0}^{*}$, the size quantization energy increases, which is accompanied by a suppression of the electrons' mutual repulsion and accordingly by decrease in the threshold value of $E_{2} / E_{d}$. Thus, in QD with $R_{0}^{*} \leq 1$, the condition for emergence of the two-electron bound state is much more favorable in comparison with the bulk semiconductor case (compare curves 3 and 4 in Fig. 1).

\section{Coefficient of the light absorption under photoionization of the two-electron impurity centers in a quasi-zero-dimensional structure}

Atoms of helium and helium-like ions in the ground state are the simplest systems in which double photoionization by a single photon can be observed. The double photoionization process under consideration is an optical transition of two electrons from bound states to the dimensionally - quantized QD states due to absorption of a photon by an impurity atom. The fundamental role of this process consists in the possibility of the theoretical study of an electron's correlations in the double photoionization spectra. Study of such reaction in semiconductive nanostructures is of particular interest in connection with the new physical situation due to dimensional quantization. In this section, the photoionization process of the two-electron impurity center, located in the semiconductive QD ground state, has been considered [5].

In this section, we consider the process of photoionization of a two-electron impurity center in the ground state in a semiconductor QD [5]:

$$
\Psi\left(\rho_{1}, \rho_{2}\right)=\frac{2^{2} \eta^{-2}}{\left(\tanh \left(R_{0}^{*} \eta^{-1}\right)-R_{0}^{*} \eta^{-1} \csc \left(R_{0}^{*} \eta^{-1}\right)\right)^{2}} \frac{\sinh \left(R_{0}^{*} \eta^{-1}-\rho_{1} \eta^{-1}\right) \sinh \left(R_{0}^{*} \eta^{-1}-\rho_{2} \eta^{-1}\right)}{\rho_{1} \rho_{2} \sinh ^{2}\left(R_{0}^{*} \eta^{-1}\right)} .
$$

The wave function of the final state will be determined by the product of the wave functions of electrons in a spherical QD:

$$
\Phi\left(\rho_{1}, \rho_{2}\right)=\Psi_{n, l, m}\left(\rho_{1}, \phi_{1}, \theta_{1}\right) \Psi_{n, l, m}\left(\rho_{2}, \phi_{2}, \theta_{2}\right),
$$


where

$$
\Psi_{n, l, m}\left(\rho_{i}, \phi_{i}, \theta_{i}\right)=\frac{\sqrt{2}}{R_{0} \sqrt{\rho_{i}}} \frac{J_{l+1 / 2}\left(k_{n l} \rho_{i}\right)}{J_{l+3 / 2}\left(\xi_{n l}\right)} Y_{l m}\left(\phi_{i}, \theta_{i}\right),
$$

here $i=1,2 ; Y_{l, m}$ - normalized ball functions; $l, m$ - orbital and magnetic quantum numbers; $J_{\nu}(x)-$ the first-kind Bessel function of the $\nu$-th order; $k_{n l}=\xi_{n l} / R_{0} ; \xi_{n l}-n$-th root of the Bessel function with $l$-th order.

The energy of one-electron states, unperturbed by impurities in a spherical QD, will have the form:

$$
E_{n, l}=\frac{\hbar^{2} \xi_{n l}^{2}}{2 m^{*} R_{0}^{2}}
$$

The effective interaction Hamiltonian with the light wave field with a unit polarization vector $\mathbf{e}_{\lambda}$ and a wave vector $\mathbf{q}$ is determined by the expression

$$
\mathbf{H}_{i n t}=-i \lambda_{0} \hbar\left(\frac{2 \pi \hbar^{2} \alpha^{*}}{\varepsilon \omega m^{* 2}} I_{0}\right)^{1 / 2} \exp (i \mathbf{q r})\left(\mathbf{e}_{\lambda} \nabla_{\mathbf{r}}\right),
$$

where $\lambda_{0}$ - the local field coefficient taking into account the difference between the amplitudes of the local and average macroscopic fields; $I_{0}$ - the light intensity; $\omega$ - the absorbed light frequency; $\epsilon$ - the static dielectric permeability of the QD material; $\alpha^{*}-$ the fine structure constant taking into account dielectric permeability.

The matrix element that determines the magnitude of the oscillator strength for the dipole optical transitions of electrons from the ground state of the two-electron impurity center (15) to the states $\Psi_{n, l, m}(\rho, \phi, \theta)$ of the discrete spectrum of quantum dots, is written as follows:

$$
\begin{array}{r}
M=i \lambda_{0} \sqrt{\frac{2 \pi \alpha^{*}}{\omega} I_{0}}\left[\left(E_{n, l, m}-E_{1}\right)\left\langle\Psi_{n, l, m}^{*}\left(\rho_{1}, \theta_{1}, \phi_{1}\right) \psi^{*}\left(\rho_{2}\right)\left|\mathbf{e}_{\lambda}, \mathbf{r}_{1}\right| \Psi\left(\rho_{1}, \rho_{2}\right)\right\rangle+\right. \\
\left.\left(E_{n, l, m}-E_{2}\right)\left\langle\Psi_{n, l, m}^{*}\left(\rho_{1}, \theta_{1}, \phi_{1}\right) \psi^{*}\left(\rho_{2}\right)\left|\mathbf{e}_{\lambda}, \mathbf{r}_{2}\right| \Psi\left(\rho_{1}, \rho_{2}\right)\right\rangle\right] .
\end{array}
$$

Taking into account (15) - (18), the expression (20) for the square of the matrix element can be written as:

$$
\begin{aligned}
|M|^{2}= & \lambda_{0}^{2} \frac{2 \pi \alpha^{*}}{\omega} I_{0} \frac{E_{d}^{2}}{\sinh ^{6}\left(R_{0}^{*} \eta^{-1}\right)} \frac{2}{a_{d}^{2} R_{0}^{* 2}\left|J_{l+3 / 2}\left(\xi_{n l}\right)\right|^{2}} \frac{2^{3} \eta^{-3}}{\left(\tanh \left(R_{0}^{*} \eta^{-1}\right)-R_{0}^{*} \eta^{-1} \csc \left(R_{0}^{*} \eta^{-1}\right)\right)^{3}} \times \\
& \mid\left[\sqrt{k_{n l}+i \eta^{-1}}\left(k_{n l}^{2}\left(R_{0}^{*}\right)^{-2}+\frac{\left|E_{1}\right|}{E_{d}}+\frac{\left|E_{2}\right|}{E_{d}}\right)^{2} k_{n l}^{-3}\left(k_{n l}^{2}+\eta^{-2}\right) \cosh \left(R_{0}^{*} \eta^{-1}\right) S\left(\sqrt{\frac{2}{\pi} R_{0}^{*}\left(k_{n l}-i \eta^{-1}\right)}\right)+\right. \\
& i \sqrt{k_{n l}-i \eta^{-1}}\left(k_{n l}+i 2 \eta^{-1}\right) \cosh \left(R_{0}^{*} \eta^{-1}\right) \operatorname{Si}\left(\sqrt{\frac{2}{\pi} R_{0}^{*}\left(k_{n l}+i \eta^{-1}\right)}\right)+ \\
& \sqrt{k_{n l}+i \eta^{-1}}\left(k_{n l}-i 2 \eta^{-1}\right) \sinh \left(R_{0}^{*} \eta^{-1}\right) \mathrm{Ci}\left(\sqrt{\frac{2}{\pi} R_{0}^{*}\left(k_{n l}-i \eta^{-1}\right)}\right)+ \\
& \left.\sqrt{k_{n l}-i \eta^{-1}}\left(k_{n l}+i 2 \eta^{-1}\right) \sinh \left(R_{0}^{*} \eta^{-1}\right) \mathrm{Ci}\left(\sqrt{\frac{2}{\pi} R_{0}^{*}\left(k_{n l}+i \eta^{-1}\right)}\right)\right]\left.\right|^{2} \times \\
\frac{2}{\pi k_{n l}}\left(\eta^{-2}+k_{n l}^{2}\right)^{-4} \mid\left\{\eta^{-1}\left[\left(\eta^{-2}+k_{n l}^{2}\right) R_{0}^{*} \cos \left(k_{n l} R_{0}^{*}\right)-\left(\eta^{-2}+2 k_{n l}+k_{n l}^{2}\right) R_{0}^{*} \sin \left(k_{n l} R_{0}^{*}\right)\right]+\right. & \left.\left(\eta^{-2}\left(k_{n l}-1\right)+k_{n l}^{2}\left(k_{n l}+1\right)\right) \sinh \left(R_{0}^{*} \eta^{-1}\right)\right\}\left.\right|^{2}
\end{aligned}
$$

here $\mathrm{Ci}(x)$ and $\mathrm{Si}(x)$ - integral cosine and sine, respectively. 
We assume that the size dispersion of QDs $u$ arises during the phase decay of a supersaturated solid solution and is satisfactorily described by the Lifshitz-Slezov formula:

$$
P(u)= \begin{cases}\frac{3^{4} e u^{2} \exp [-1 /(1-2 u / 3)]}{2^{5 / 3}(u+3)^{7 / 3}(3 / 2-u)^{11 / 3}}, & u<3 / 2 \\ 0, & u>3 / 2\end{cases}
$$

where $u=R_{0} / \bar{R}_{0}, R_{0}$ and $\bar{R}_{0}$ - the QD radius and its mean value, correspondingly; $e$ - base of the natural logarithm.

The light impurity absorption coefficient $K(\omega)$, taking into account dispersion of the QD size, is determined by the expression:

$$
K(\omega)=\frac{2 \pi N_{0}}{\hbar I_{0}} \sum_{n} \int_{0}^{3 / 2} d u P(u)|M|^{2} \delta\left(E_{n, 0,1}+\left|E_{1}\right|+\left|E_{2}\right|-\hbar \omega\right),
$$

where $N_{0}$ - the QD concentration in dielectric matrix; $\delta(x)$ - the Dirac Delta function.

Taking into account (21) and performing integration in (23), the light impurity absorption coefficient $K(\omega)$ can be written in the next form:

$$
\begin{aligned}
& K(X)= \\
& \sum_{n=1}^{N} P\left(u_{n}\right) \frac{2^{3} \lambda_{0}^{2} \pi \alpha^{*}}{X} \times \frac{E_{d}\left(\eta^{-2}+\xi_{n l}^{2}\left(\bar{R}_{0}^{*}\right)^{-2} u_{n}^{-2}\right)^{-6}}{a_{d}^{2}\left(\bar{R}_{0}^{*}\right)^{2} u_{n}^{2} \sinh ^{6}\left(\bar{R}_{0}^{*} u_{n} \eta^{-1}\right) \pi \xi_{n l}\left(\bar{R}_{0}^{*}\right)^{-1} u_{n}^{-1}\left|J_{l+3 / 2}\left(\xi_{n l}\right)\right|^{2}} \times \\
& \frac{2^{3} \eta^{-3}}{\left(\tanh \left(\bar{R}_{0}^{*} u_{n} \eta^{-1}\right)-\bar{R}_{0}^{*} u_{n} \eta^{-1} \csc \left(-\bar{R}_{0}^{*} u_{n} \eta^{-1}\right)\right)^{3}}\left(2 \xi_{n l}^{2}\left(\bar{R}_{0}^{*}\right)^{-2} u_{n}^{-2}+\left|E_{1}\right| / E_{d}+\left|E_{2}\right| / E_{d}\right)^{2} \xi_{n l}^{3}\left(\bar{R}_{0}^{*}\right)^{-3} u_{n}^{-3} \times \\
& \mid \sqrt{\xi_{n l} \bar{R}_{0}^{*}+i \eta^{-1}}\left(\xi_{n l} \bar{R}_{0}^{*}-i 2 \eta^{-1}\right) \cosh \left(\bar{R}_{0}^{*} u_{n} \eta^{-1}\right) \operatorname{Si}\left(\sqrt{\frac{2}{\pi} \bar{R}_{0}^{*} u_{n}\left(\xi_{n l}\left(\bar{R}_{0}^{*}\right)^{-1} u_{n}^{-1}-i \eta^{-1}\right)}\right)+ \\
& i \sqrt{\xi_{n l}\left(\bar{R}_{0}^{*}\right)^{-1} u_{n}^{-1}-i \eta^{-1}}\left(\xi_{n l}\left(\bar{R}_{0}^{*}\right)^{-1} u_{n}^{-1}+i 2 \eta^{-1}\right) \cosh \left(\bar{R}_{0}^{*} u_{n} \eta^{-1}\right) \times \\
& \mathrm{Si}\left(\sqrt{\frac{2}{\pi} \bar{R}_{0}^{*} u_{n}\left(\xi_{n l}\left(\bar{R}_{0}^{*}\right)^{-1} u_{n}^{-1}+i \eta^{-1}\right)}\right)+ \\
& \sqrt{\xi_{n l}\left(\bar{R}_{0}^{*}\right)^{-1} u_{n}^{-1}-i \eta^{-1}}\left(\xi_{n l}\left(\bar{R}_{0}^{*}\right)^{-1} u_{n}^{-1}-i 2 \eta^{-1}\right) \sinh \left(\bar{R}_{0}^{*} u_{n} \eta^{-1}\right) \times \\
& \mathrm{Ci}\left(\sqrt{\frac{2}{\pi} \bar{R}_{0}^{*} u_{n}\left(\xi_{n l}\left(\bar{R}_{0}^{*}\right)^{-1} u_{n}^{-1}-i \eta^{-1}\right)}\right)+ \\
& \sqrt{\xi_{n l}\left(\bar{R}_{0}^{*}\right)^{-1} u_{n}^{-1}-i \eta^{-1}}\left(\xi_{n l}\left(\bar{R}_{0}^{*}\right)^{-1} u_{n}^{-1}+i 2 \eta^{-1}\right) \sinh \left(\bar{R}_{0}^{*} u_{n} \eta^{-1}\right) \times \\
& \left.\mathrm{Ci}\left(\sqrt{\frac{2}{\pi} \bar{R}_{0}^{*} u_{n}\left(\xi_{n l}\left(\bar{R}_{0}^{*}\right)^{-1} u_{n}^{-1}+i \eta^{-1}\right)}\right)\right|^{2} \times \\
& \mid \eta^{-1}\left[\left(\eta^{-2}+\xi_{n l}^{2}\left(\bar{R}_{0}^{*}\right)^{-2} u_{n}^{-2}\right) \bar{R}_{0}^{*} u_{n} \cos \left(\xi_{n l}\right)-\bar{R}_{0}^{*} u_{n} \sin \left(\xi_{n l}\right)\left(\eta^{-2}+2 \xi_{n l}\left(\bar{R}_{0}^{*}\right)^{-1} u_{n}^{-1}+\xi_{n l}^{2}\left(\bar{R}_{0}^{*}\right)^{-2} u_{n}^{-2}\right)\right]+ \\
& \left.\left(\eta^{-2}\left(\xi_{n l}\left(\bar{R}_{0}^{*}\right)^{-1} u_{n}^{-1}-1\right)+k_{n l}^{2}\left(\xi_{n l}\left(\bar{R}_{0}^{*}\right)^{-1} u_{n}^{-1}+1\right)\right) \sinh \left(\bar{R}_{0}^{*} u_{n} \eta^{-1}\right)\right|^{2}
\end{aligned}
$$

where $u_{n}=\xi_{n l}^{2} /\left(\left(\bar{R}_{0}^{*}\right)^{2}\left(X-\left|E_{1}\right| / E_{d}-\left|E_{2}\right| / E_{d}\right)\right) ; N=[n]-$ is an integral part of the solution for a transcendental equation of the form: $\xi_{n l}^{2}=3\left(\bar{R}_{0}^{*}\right)^{2}\left(X-\left|E_{1}\right| / E_{d}-\left|E_{2}\right| / E_{d}\right) / 2$.

Figures 2( $\mathrm{a}$ and $\mathrm{b}$ ) show the calculated double photoionization spectra, the characteristic feature of which (see curve 1 in Fig. 2(a) and curves $1-3$ in Fig. 2(b)) is the two-humped profile due to electron correlations. Distance between the spectral curve maxima is determined by modulus of the difference between the first and second ionization potentials of the two-electron impurity center. Fig. 2(a) shows that as the second ionization potential increases, the impurity absorption edge shifts to the spectrum short-wavelength region and the right-hand peak transforms at first to the step (curve 2 in Fig. 2(a)), and then completely disappears on the spectral curve (see curve 3 in Fig. 2(a)). Fig. 2(b) 

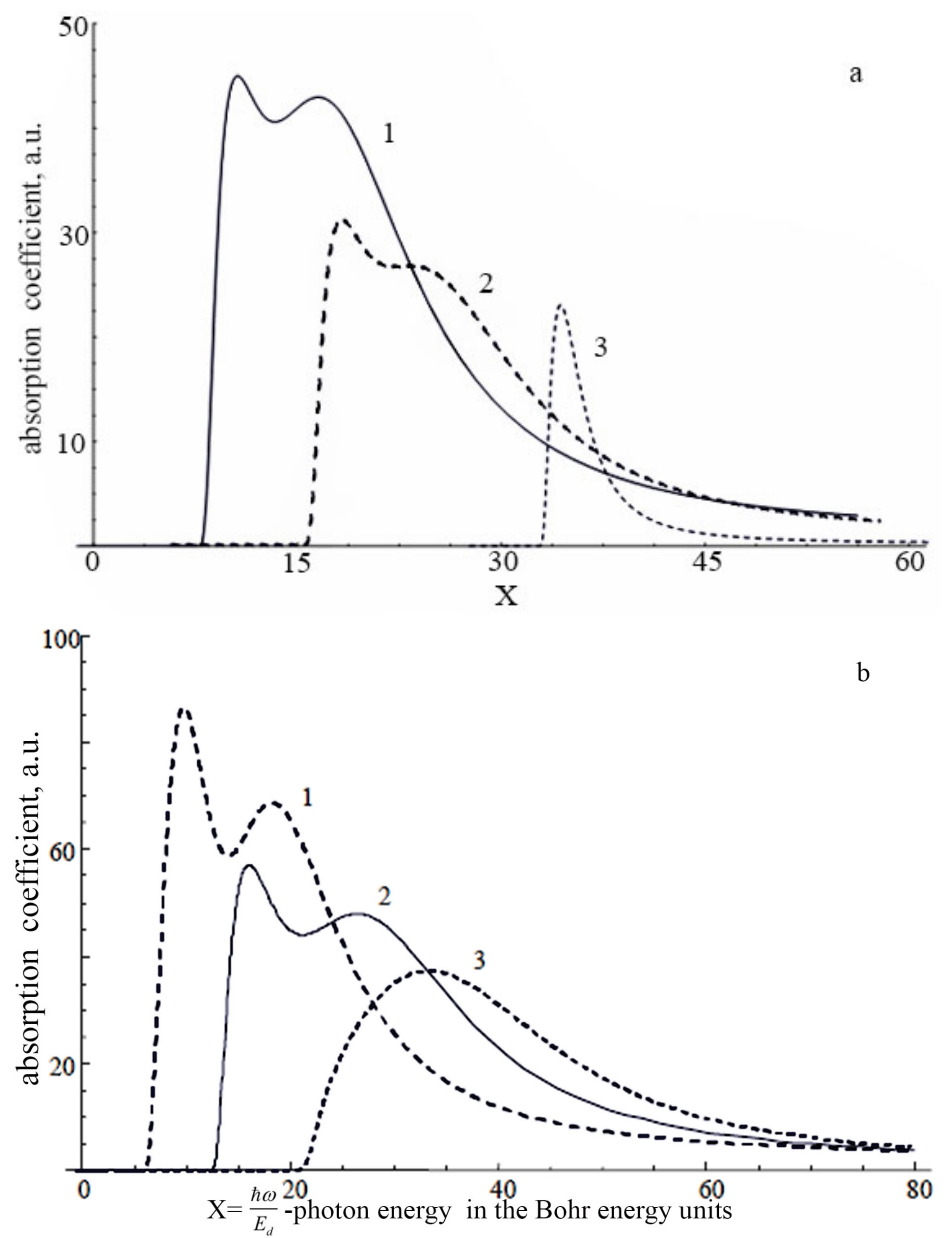

FIG. 2. Spectral dependence of the light absorption coefficient for double photoionization of the two-electron impurity centers in a quasi-zero-dimensional structure: a) for different values of the second ionization potential $E_{2}$ :

1: $E_{2}=0.04 \mathrm{eV} ; 2: E_{2}=0.05 \mathrm{eV} ; 3: E_{2}=0.08 \mathrm{eV}$;

b) for different values of $R_{0}^{*}: 1-3 ; 2-1 ; 3-0.5$, with $E_{2}=0.04 \mathrm{eV}$

shows transformation of the right peak on the spectral curve with a decrease in the QD mean radius $\bar{R}_{0}^{*}$, as a result we can see, that this peak disappears. This is due to the fact that the dimensionally - quantization energy increases with decreasing of $\bar{R}_{0}^{*}$, as a result of which the electronic correlation is suppressed.

\section{Conclusions}

Generalization of the zero-range potential method to the case of two-electron impurity centers in a QD has been carried out. The first ionization potential has been calculated by the variational method, within the semiempirical model of a two-electron impurity center in the spherically symmetric QD. It is shown, that, unlike the case of a bulk semiconductor in QD, formation of the two-electron bound states is possible at sufficiently low ionization potential values, as well as for the nucleus zero charge of an impurity center. Diamagnetic susceptibility of the two-electron and one-electron impurity centers has been calculated for the semiconductive QD. It is shown that an increase in the QD radius leads to an increase in the diamagnetic susceptibility value, which is associated with an increase in the localized state radius. A comparison of the diamagnetic susceptibility for quasi-zero-dimensional two-electron impurity centers and for $D^{-}$-centers shows that in case of the two-electron impurity centers, the diamagnetic susceptibility is several times larger. In the dipole approximation, in the framework of the effective-mass method, the light impurity absorption coefficient has been calculated for a double ionization of the two-electron impurity center by a single photon. It is shown that a characteristic feature of the double photoionization spectrum is the spectral curve's "two-humped" profile. The distance between the spectral curve maxima is determined by the difference between the first and second ionization potentials of the two-electron impurity center. 


\section{Acknowledgements}

This work was supported by the Ministry of Education and Science of the Russian Federation (projects No. 3.6321.2017/8.9 and 2019-0940).

\section{References}

[1] Grinberg A.A., Belorusets E.D. On the energy spectrum of multiply charged impurity centers in semiconductors. Solid State Physics, 1978, 20, P. 1970-1978.

[2] Lucovsky G. On the photoionization of deep impurity centers in semiconductors. Solid State Communications, $1965, \mathbf{3}, 299$.

[3] Belorusets E.D., Imamov E.Z., Mamatkulov B.R. Diamagnetism of two-electron deep impurity centers in semiconductors. Physics and technology of semiconductors, 1982, 16 (1),89.

[4] Imamov E.Z., Krevchik V.D. On Diamagnetism of Deep Impurities in Quantized Semiconductor Film. Phys. St. Sol.(b), 1982, 114,201.

[5] Krevchik V.D., Levashov A.V. Energy spectrum and optical properties of the complex "quantum dot - impurity center". Physics and technology of semiconductors, 2002, $36(2), 216$.

[6] Pakhomov A.A., Khalipov K.V., Yassievich I.N. Local electronic states in semiconductor quantum wells. Physics and technology of semiconductors, 1996, 30 (8), 1387.

[7] Mikhailov A.I., Mikhailov I.A. Double photoionization and ionization with excitation under the Compton scattering of high-energy photons on metastable states of the helium-like ions. JETP, 1999, 116 (6), 1889.

[8] Eseev M.K., Matveev V.I. Investigation of the analytic wave functions for two-electron systems under dynamic interactions with the multiply charged ions and with the ultrashort pulses of an electromagnetic field. Journal of technical physics, 2008,78 (8),28.

[9] Zaytsev S.A., Knyr V.A., Popov Yu.V., Lahmam-Bennani A. Application of the J-matrix method to Faddeev-Merkuriev equations for (e,2e) reactions: Beyond pseudo-states. Physical Review A, 2007, 75 (2),022718.

[10] Pandey R.K., Harbola Manoj K., Singh Vijay A. Helium-like donors in semiconductor quantum dots. J. Phys.: Condens. Matter, 2004, 16,1769.

[11] Castro Arata P.A. Two-electron impurity centers as a tool for the chalcogenide glassy semiconductors studying (St. Petersburg: Publishing House of the Russian State Pedagogical University named by A. I. Herzen), 2006.

[12] Boyarkina N.I., Vasil'ev A.V. Participation of the crystal electronic subsystem in reactions between multiply charged centers in semiconductors. Physics and technology of semiconductors, 2000, 34 (2), 172.

[13] Petrov P.V., Ivanov Yu.L., Averkiev N.S. The photoluminescence circular polarization of the two-dimensional system with $A^{+}$-centers in an external magnetic field. Physics and technology of semiconductors, 2011, 45 (27), 794.

[14] Huant S., Mandray A., et al. Well-width dependence of D-cyclotron resonance in quantum wells. Physical Review B, $1993, \mathbf{4 8 , 2 3 7 0 . ~}$ 